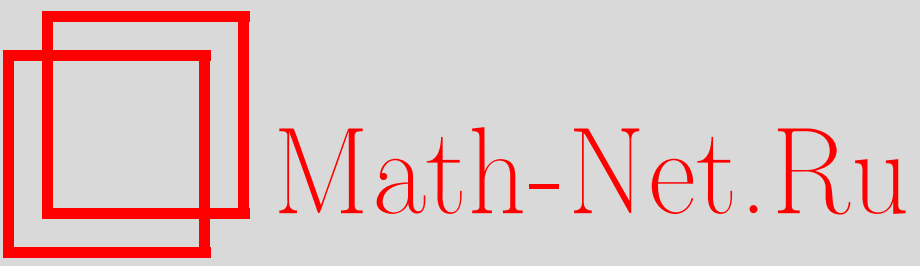

О. А. Олейник, Г. Каллианпур, О задачах со свободной границей, возникающих в теории вероятностей (теоремы существования), УМН, 1997, том 52, выпуск $1,229-230$

DOI: https://doi.org/10.4213/rm811

Использование Общероссийского математического портала Math-Net.Ru подразумевает, что вы прочитали и согласны с пользовательским соглашением

http: //www. mathnet.ru/rus/agreement

Параметры загрузки:

IP : 3.85 .5 .30

26 апреля 2023 г., 16:29:59 


\title{
О ЗАДАЧАХ СО СВОБОДНОЙ ГРАНИЦЕЙ, ВОЗНИКАЮЩИХ В ТЕОРИИ ВЕРОЯТНОСТЕЙ (ТЕОРЕМЫ СУЩЕСТВОВАНИЯ)
}

\author{
Г. КАЛЛИАНПУР, О. А. ОЛЕЙнИК
}

В [1] рассмотрены задачи со свободной границей, возникающие при изучении вопроса об оптимальной остановке марковского процесса, и доказаны теоремы единственности решений таких задач. Здесь мы докажем соответствующие теоремы существования решения. При этом мы используем методы, близкие к предложенным в [2], [3].

Рассматриваемая здесь задача со свободной границей сформулирована в [1], и там же указано интегральное тождество, определяющее слабое решение задачи.

Заметим, что задача со свободной границей (1)-(4) из [1] может иметь бесконечное число решений, если мы расширим указанные там классы единственности. Приведем пример. Пусть

$$
\begin{gathered}
\frac{\partial u}{\partial t}+\frac{\partial^{2} u}{\partial x^{2}}=0 \text { в } Q=\{t, x: 0<t<\infty, 0<x<1\} \\
\left.u\right|_{x=0}=0,\left.\quad u\right|_{x=1}=0,\left.\quad u\right|_{x=x^{*}}=0,\left.\quad \frac{\partial u}{\partial x}\right|_{x=x^{*}-0}-\left.\frac{\partial u}{\partial x}\right|_{x=x^{*}+0}=0 .
\end{gathered}
$$

Здесь свободной границей является прямая $x=x^{*}, \lambda(t, x) \equiv 0$.

Пусть $\Lambda_{1}, \Lambda_{2}, \ldots$ - последователность собственных значений задачи

$$
\frac{d^{2} v_{j}}{d x^{2}}+\Lambda_{j} v_{j}=0, \quad v_{j}(0)=0, \quad v_{j}(1)=0,
$$

$v_{j}(x)$ - соответствующие собственные функции. При $j>1$ существует $x_{j}$ такое, что $0<x_{j}<1$ и $v_{j}\left(x_{j}\right)=0$. Очевидно, что функция $u_{j}(t, x)=\exp \left\{\Lambda_{j} t\right\} v_{j}(x), j=2,3, \ldots$, является решением задачи $(1),(2)$ с $x^{*}=x_{j}$ и $u_{j}(t, x) \rightarrow \infty$ при $t \rightarrow \infty$, если $x \neq x^{\prime}$ и $v_{j}\left(x^{\prime}\right)=0$, так как $\Lambda_{j}>0$ при всех $j$.

Имеет место следующая теорема существования слабого решения задачи (1)-(4), определенной в [1]. Мы пользуемся обозначениями работы [1].

Tеорема. 1) Пусть $k(t, x, u) c(t, x, u) \leqslant \gamma_{0}=$ const $<0$ npu $t>T$. Тогда существует ограниченное в $Q$ слабое решение задачи (1)-(4) из [1]. Ecли $f(t, x, u) \equiv 0$ при $t>T, T-$ некоторая постоянная, $u\left|u_{1}(t)\right|+\left|u_{2}(t)\right| \leqslant C_{1} e^{-\alpha_{1} t}$ nри $t>T, C_{1}, \alpha_{1}=$ const $>0$, mо существует слабое решение $и(t, x)$ задачи (1)-(4) из [1], удовлетворяющее условию

$$
|u(t, x)| \leqslant C_{2} e^{-\alpha_{2} t}, \quad C_{2}, \alpha_{2}=\text { const }>0 .
$$

2) Пусть $k(t, x, u) c(t, x, u) \leqslant \gamma_{1}, \gamma_{1}=$ const $>0$. Тогда существует слабое решение задачи (1)-(4) из [1] такое, что

$$
|u(t, x)| \leqslant C_{3} e^{\alpha_{3} t}, \quad C_{3}, \alpha_{3}=\text { const }>0,
$$

где $\alpha_{3}$ зависит от $\gamma_{1}$, если $\left|u_{1}(t)\right|+\left|u_{2}(t)\right| \leqslant C_{4} e^{-\alpha_{4} t}$ и $\alpha_{4}>0$ - достаточно большая постоянная, $f(t, x, u) \equiv 0$ при $t>T$.

3) $E$ сли $k(t, x, u) c(t, x, u) \leqslant 0 u|k(t, x, u) b(t, x, u)| \leqslant \beta$, где $\beta$ - достаточно малая постоянная, то существует слабое решение задачи (1)-(4) из [1], удовлетворяющее условию

$$
|u(t, x, u)| \leqslant C_{5} e^{-\alpha_{5} t}, \quad C_{5}, \alpha_{5}=\text { const }>0,
$$

$n p u\left|u_{1}(t)\right|+\left|u_{2}(t)\right|+|f(t, x, u)| \leqslant C_{6} e^{-\alpha_{6} t}, C_{6}, \alpha_{6}=$ const $>0$, и $\alpha_{6}$ - достаточно большая постоянная. Если $k(t, x, u) c(t, x, u) \leqslant 0$ в $Q$ u $f(t, x, u) \equiv 0$ при $t>T$, то существует ограниченное в $Q$ слабое решение задачи (1)-(4) из [1]. 


\section{СПИСОК ЛИТЕРАТУРЫ}

[1] Каллианпур Г., Олейник О. А. // УМН. 1996. Т. 51. №6. С. 205-206. [2] Олейник О. А. // ДАН СССР. 1960. Т. 135. № 5. С. 1054-1058. [3] Oleinik O. A. On Stefan-type free boundary problems for parabolic equations // In Seminari 1962-1963 di analisi, algebra, geometria e topologia 1. Roma, 1965. P. 388-403.

Московский государственньй университет им. М. В. Ломоносова;

Математический институт

им. В. А. Стеклова РАН
Принято редколлегией 23.10.1996 